\title{
Multistep reactions using microreactor chemistry
}

\author{
Batoul Ahmed-Omer, ${ }^{\text {a,b }}$ David A. Barrow, ${ }^{a}{ }^{*}$ and Thomas Wirth ${ }^{\mathrm{b}} *$ \\ ${ }^{a}$ Laboratory for Applied Microsystems, Cardiff School of Engineering, Cardiff University, \\ Cardiff CF24 3AA, United Kingdom \\ ${ }^{b}$ School of Chemistry, Cardiff University, Park Place, Cardiff CF10 3AT, United Kingdom
}

E-mails: barrow@cf.ac.uk,wirth@cf.ac.uk

\section{Dedicated to Professor Siegfried Blechert on the occasion of his $65^{\text {th }}$ birthday}

\begin{abstract}
A microflow system for the investigation of multistep syntheses involving Pd-catalysed Heck reactions and Ru-catalysed ring-closing metathesis is described. A successful provision of reagent and catalyst delivery in a consecutive fashion allowed control over reaction parameters leading to fast optimisation. The performance of Heck reactions in multistep procedures was not as successful as in the single step coupling, whereas the ring-closing metathesis proved to be successful in the production of the desired compounds for further functionalisation.
\end{abstract}

Keywords: Catalysis, Heck reaction, metathesis, microreactors, multistep synthesis

\section{Introduction}

Consecutive multistep synthesis using microflow techniques offer advantages over conventional methods. In addition to a better control over reaction parameters and fast optimisation, multistep reactions in flow enable an improved and more controlled addition of reagents in a consecutive fashion which can be accomplished without delays. It is indeed the continuous nature of the system that allows efficient reaction optimisation: small aliquots of product can be collected as they are produced, and analysed in order to decide how to change reaction conditions and parameters without stopping the process. The efficiency and speed of optimisation could be further improved by introducing automation in addition to fast online analysis into the systems allowing the chemist to obtain faster feedback. There is a growing interest in the literature in carrying out consecutive multistep syntheses using microflow technique by exploiting the above mentioned advantages. ${ }^{1}$ 


\section{Results and Discussion}

The typical multistep microflow setup used in this investigation consisted of two or more separate microflow sections connected to each other. In a two-step microflow setup, the first reaction step takes place in section 1 where the compounds $\mathbf{A}$ and $\mathbf{B}$ mix and react to form intermediate $\mathbf{C}$ as illustrated in Figure 1. Once the intermediate is formed, the second reaction step takes place after introducing the required reagent $\mathbf{D}$ before entering section 2 to form product $\mathbf{E}$. Product $\mathbf{E}$ is then collected at the output of section 2. A coiled polytetrafluoroethylene (PTFE) microtubing was used along with multi-way connectors enabling an easy variation of different sections, or introduce reagents as required, with good flexibility. A limitation with the multistep setup is that once the required residence time for section 1 has been set, the total flow rate in section 2 becomes fixed and the residence time in section 2 can only be changed by varying the length of the section.

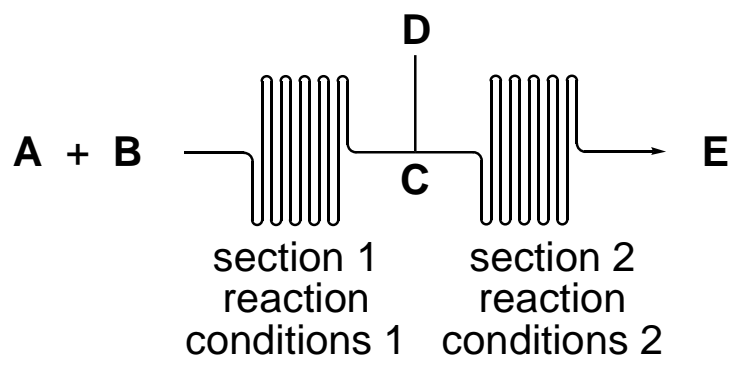

Figure 1. Multistep reactions using flow chemistry.

We already have reported on simple Heck reactions using flow chemistry. ${ }^{2}$ Also other research groups have been investigated Heck reactions in flow using supported palladium catalysts. Kirschning et $a .^{3}$ used a monolith containing nanoparticular palladium while Garcia-Verdugo and Luis et al. ${ }^{4}$ investigated an imidazolium-functionalized polystyrene monolith successfully for such cross-coupling reactions. Palladium-containing monoliths were also used by Ley et al. for Heck reactions carried out in superheated ethanol. ${ }^{5}$ Seeberger and co-workers used palladium on charcoal as a catalyst for Heck reactions. ${ }^{6}$ Jensen and Buchwald demonstrated an acceleration of such reactions when performed at elevated temperatures and pressures facilitated by microreactors ${ }^{7}$ and used a similar reaction for automated optimisation. ${ }^{8}$ A review on the use of transition metalcatalyzed Heck reactions using microwave and microreactor technologies has appeared recently. ${ }^{9}$

Our interest focused on carrying out a multistep microflow synthesis consisting of a combination of a ruthenium-catalysed ring closing metathesis (RCM) followed by a Heck coupling reaction. Precursor 1 was prepared by allylation of $o$-iodoaniline and then subjected to a sequence of metathesis and Heck reaction. In the first step of the reaction sequence, product 2 was prepared by metathesis of $N, N$-diallyl-2-iodoaniline 1 and isolated in $98 \%$ yield, with only traces of the side product 1-(2-iodophenyl)-1H-pyrrole 3. 


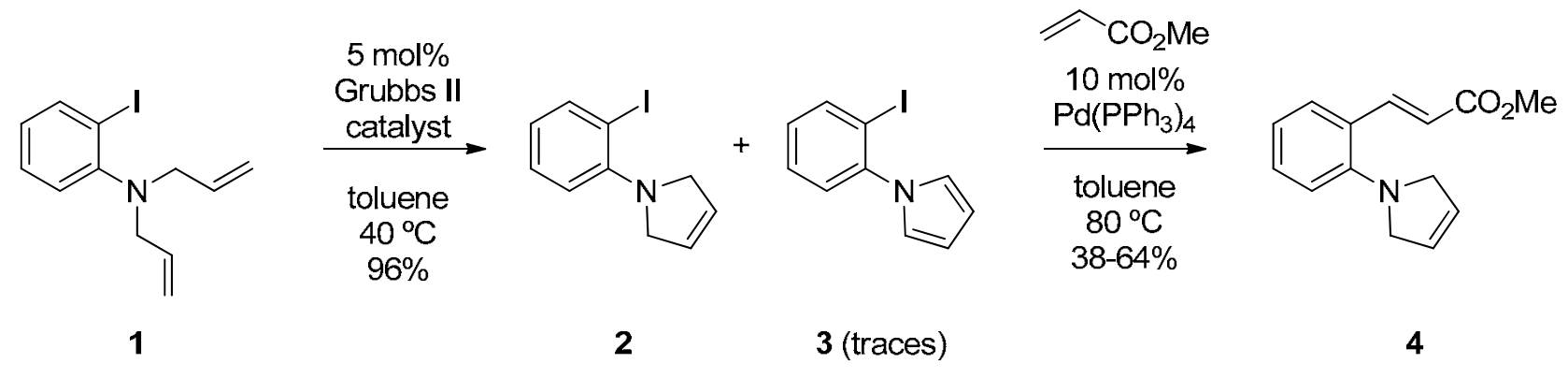

Scheme 1. Metathesis and Heck reaction of diallylaniline 1.

A combination of the metathesis reaction with a Heck reaction as the second step was carried out using methyl acrylate. After a series of optimisation experiments, $(E)$-methyl 3-(2-(2H-pyrrol$1(5 H)$-yl) phenyl) acrylate 4 was obtained in yields between $38 \%$ and $64 \%$. When the Heck reaction was carried out using less activated substrates such as styrene, $p$-fluorostyrene, $p$ trifluoromethylstyrene and $m$-nitrostyrene, no coupling product was observed.

A similar example of a consecutive RCM and Heck reaction was carried out using the metathesis precursor $N, N^{\prime}$-diallylaniline 5, prepared in $89 \%$ yield from aniline. The RCM of compound 5 was carried out in the presence of Grubbs II catalyst using the reaction setup shown in Figure 1 to afford the intermediate 1-phenyl-2,5-dihydro- $1 H$-pyrrole 6 in 96\% yield. Compound 6 was then reacted with iodobenzene and 1,3-diphenyl-1H-pyrrole 8 was obtained in yields between $53 \%$ and $60 \%$ (Scheme 2). In addition to the Heck reaction, an aromatisation of the heterocyclic ring system is occuring under the reaction conditions and the pyrrole derivative $\mathbf{8}$ is the only reaction product observed in this reaction sequence.

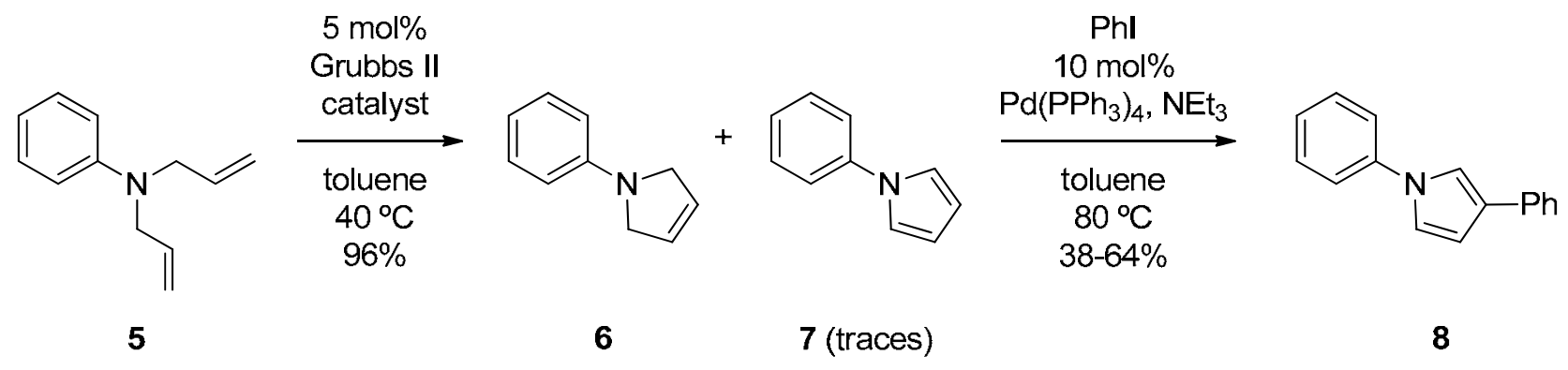

Scheme 2. Metathesis and Heck reaction sequence.

In this reaction sequence, the solvent flow was separated into a segmented flow. In such multiphase flow mass transfer is accelerated by alternating immiscible fluid packets benefiting from having both (i) a continually refreshing interface between adjacent fluid packets, and (ii) a 
rapid vortex flow within a fluid packet. ${ }^{10}$ One early study of the Heck reaction under biphasic conditions was reported using toluene/ethylene glycol as a biphasic solvent system. ${ }^{11}$ Keeping the catalyst separated from the reactants and product phase is a typical strategy used in biphasic catalysis giving the advantage of product/catalyst separation and recycling. Such a strategy has also been adopted by dissolving the palladium catalyst in an ionic liquid. This allowed Heck reactions with an easy continuous recycling of the catalyst. ${ }^{12}$

Particularly in the metathesis reaction it is very important to avoid the use of nucleophilic solvents such as acetonitrile or dimethylformamide, as they would affect the catalyst's performance. The need of carrying out the Heck reaction at high temperature seems to make toluene an ideal solvent for the two-step synthesis. However, there are very few solvents which are immiscible with toluene in order to create segmentation, and are compatible with the catalytic reactions. Water and ethylene glycol are incompatible with the reaction conditions, therefore a perfluorocarbon solvent (perfluorononane) was chosen as the inert phase to form segmentation, due to its compatibility with toluene as well as with a wide range of organic solvents in terms of immiscibility.

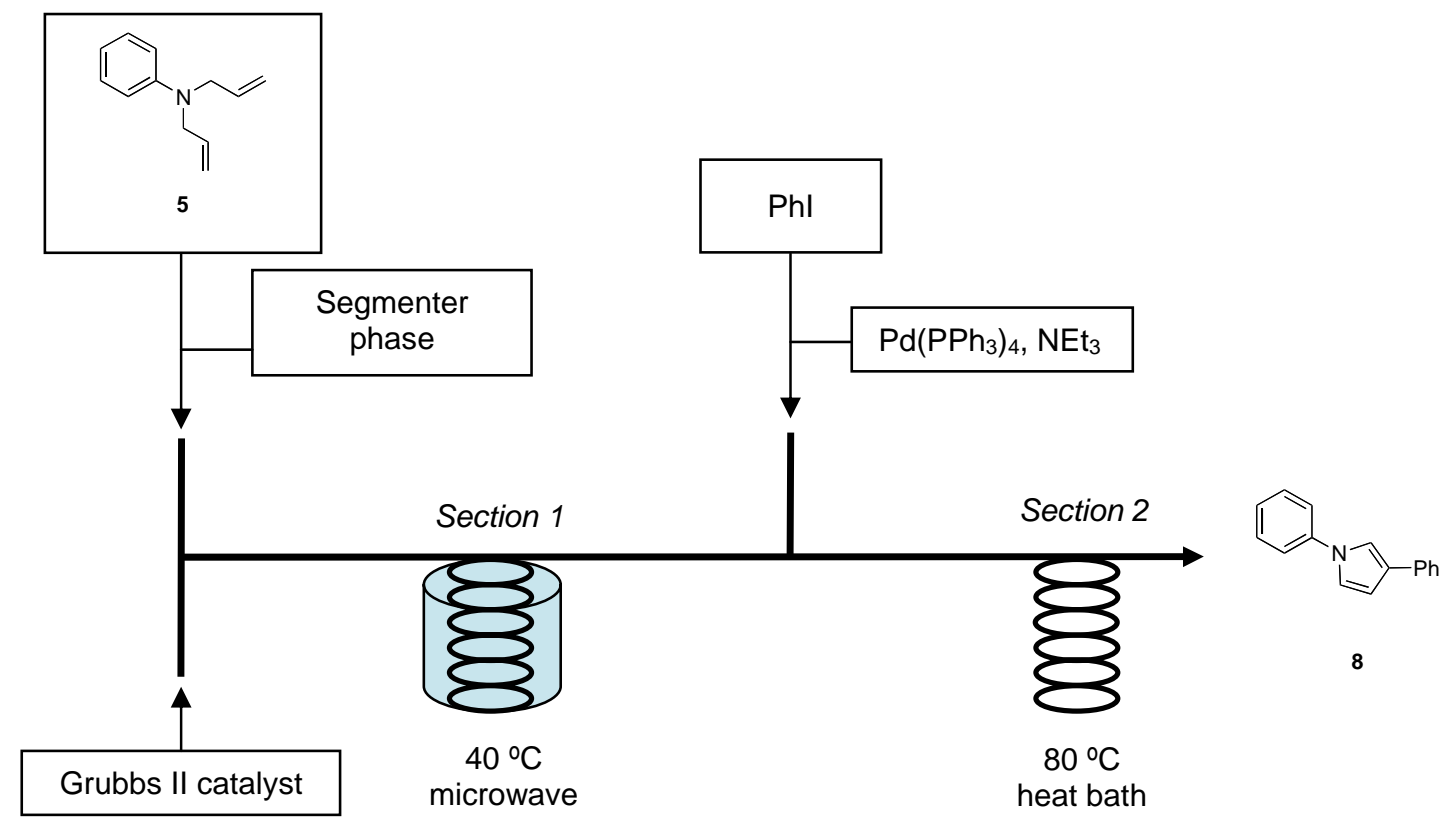

Scheme 3. Consecutive reactions in segmented flow.

A third substrate was investigated in a similar multistep sequence. $t$-Butyl-2(diallylamino)phenylcarbamate 9 was prepared in a conventional flask synthesis by $\mathrm{N}$-allylation of benzene-1,2-diamine with allyl bromide producing a mixture of five $\mathrm{N}$-allylated derivatives with the desired $N, N$-diallyl-1,2-phenylenediamine being the major product. After BOC protection the compound 9 was employed in a metathesis reaction using Grubbs catalyst ${ }^{13}$ as the first step of the microflow synthesis. The metathesis reaction was initially conducted with compound 9 using 5 
mol\% of Grubbs I catalyst in a PTFE microtube at $40{ }^{\circ} \mathrm{C}$, obtaining product $\mathbf{1 0}$ in a modest $63 \%$ yield, along with the side product $t$-butyl-2-(1H-pyrrol-1-yl)-phenylcarbamate 11 in $32 \%$ yield. In order to improve the yield of product 10, we carried out a brief optimisation study of the metathesis reaction by investigating the effect of the four main factors affecting the reaction, i.e. choice of catalyst, load of catalyst, heating method and temperature. The most significant results are summarised in Table 1.

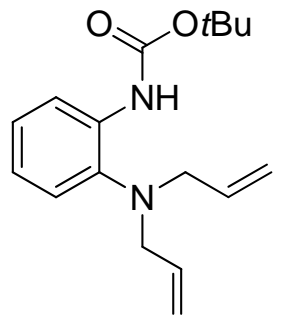

9

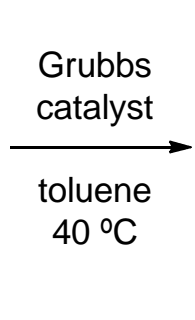

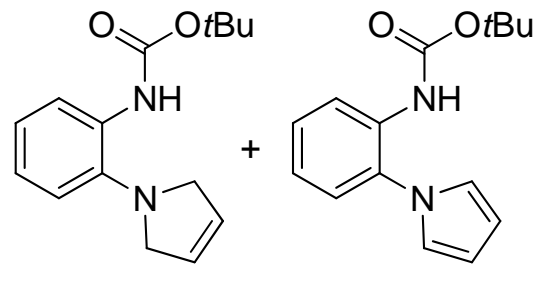

11

Scheme 4. Metathesis reaction of compound 9.

Table 1. Optimisation of RCM of $t$-butyl-2-(diallylamino)phenylcarbamate 9 in PTFE microtubing

\begin{tabular}{ccccc}
\hline Catalyst & $\begin{array}{c}\text { Amount of catalyst } \\
{[\mathrm{mol} \%]}\end{array}$ & Heating method & $\begin{array}{c}\text { Yield of 10 } \\
{[\%]}\end{array}$ & $\begin{array}{c}\text { Yield of 11 } \\
{[\%]}\end{array}$ \\
\hline Grubbs I & 1 & Oil bath & 31 & 23 \\
Grubbs I & 5 & Oil bath & 63 & 32 \\
Grubbs I & 5 & MW (150 W) & 73 & 21 \\
Grubbs II & 1 & Oil bath & 48 & 19 \\
Grubbs II & 5 & Oil bath & 87 & 16 \\
Grubbs II & 5 & MW (150 W) & 91 & 4 \\
\hline
\end{tabular}

Reaction conditions: Grubbs catalyst, compound 9, solvent system (toluene / perfluorononane), residence time 10 minutes, reaction temperature $40{ }^{\circ} \mathrm{C}$.

In general it was observed that by changing the RCM catalyst type from Grubbs I to Grubbs II the yield of the desired product $\mathbf{1 0}$ improved significantly with a decrease of the yields of side product 11. Further studies showed that catalyst loadings larger than $5 \mathrm{~mol} \%$ and an increase in 
temperature led to an increase in the yield of side product $\mathbf{1 1}$ and a decrease of the yield of compound 10. Overall, the best results were obtained by using microwave irradiation at $40{ }^{\circ} \mathrm{C}$, using Grubbs II catalyst (5 mol\%), which gave compound $\mathbf{1 0}$ in $91 \%$ yield in a very short reaction time (10 minutes). In this particular example, we found that heating by microwave irradiation led to a better reproducability than oil bath heating.

After finding the optimal metathesis conditions for compound 9, we aimed to accomplish the cyclisation of intermediate $\mathbf{1 0}$ by initially removing the carbamate protecting group using the acidic conditions of the subsequent diazotation step using of $t$-butyl nitrite and acetic acid. But the removal of the protecting group, necessary for the formation of diazonium, did not take place under the reaction conditions. The use of stronger acids caused polymerisation or decomposition / oxidation of the 2,5-dihydropyrrole moiety and compound $\mathbf{1 1}$ was obtained as the major product, along with small traces of the precursor 10. Such an oxidation is also observed to occur slowly on the isolated substrate exposed to air, while it is accelerated in the presence of Grubbs catalyst. With the substrate 9 the coupling of the metathesis reaction with a consecutive Heck reaction was, therefore, not possible.

In conclusion, we have developed a microflow system to study multistep syntheses involving Pd-catalysed Heck reactions and Ru-catalysed ring-closing metathesis. A successful provision of reagent and catalyst delivery in a consecutive fashion allowed control over reaction parameters and led to fast optimisation. The performance of Heck reactions in multistep procedures was not as successful as in the single step coupling, calling for further protocol optimisation. Ring-closing metathesis, on the other hand, proved quite successful in the production of the desired compounds for further functionalisation.

\section{Experimental Section}

\section{General procedure for allylation of arylamines}

To a solution of arylamine (1,2-phenylenediamine, $1.0 \mathrm{~g}, 9.2 \mathrm{mmol}$; o-iodoaniline, $1.0 \mathrm{~g}, 4.0$ $\mathrm{mmol}$; or aniline, $1.0 \mathrm{~g}, 10.7 \mathrm{mmol}$ ), allyl bromide (2.2 equivalents to the aniline) was added dropwise in dry DMF $(10-15 \mathrm{ml})$ at $0{ }^{\circ} \mathrm{C}$. The mixture was heated to $60{ }^{\circ} \mathrm{C}$ and stirred for 48 hours. The crude mixture was then treated with an aqueous solution of $\mathrm{NaOH}(10 \mathrm{ml}, 6.0 \mathrm{M})$ then left to stir. After approx. $1 \mathrm{~h}$ stirring, the reaction mixture was extracted once with ethyl acetate (10 $\mathrm{ml}$ ) and the organic phase dried with magnesium sulfate. The crude product was purified using chromatography to give the desired product, either $N, N$-diallyl-1,2-phenylenediamine ( $0.9 \mathrm{~g}, 55 \%$ yield) as pale yellow oil, $N, N$-diallyl-2-iodobenzenamine $1(0.93 \mathrm{~g}, 77 \%$ yield $)$ as brown oil, or $N, N$-diallylaniline $5(1.6 \mathrm{~g}, 89 \%)$ as a colourless oil.

\section{General procedure for ring closing metathesis (RCM) under segmented flow conditions in microflow}

Stock solutions were prepared in individual Schlenk tubes under inert atmosphere: (A) A 0.1 M solution of RCM substrate $(0.4 \mathrm{mmol})$ in toluene $(4.0 \mathrm{ml})$ : (B) A $0.1 \mathrm{M}$ solution of either Grubbs 
catalyst I (16.5 mg, $0.02 \mathrm{mmol})$ or Grubbs catalyst II (17.0 mg, $0.02 \mathrm{mmol})$ in toluene (0.2 $\mathrm{ml})$; (C) pure perfluorocarbon solvent (perfluorodecalin or perfluorononane) used as a segmenting phase ( 5 $\mathrm{ml}$ ). Each solution was loaded individually into a gas-tight glass syringe which was then connected onto the microflow system (PTFE) through a designated inlet using a T-connector: length 2000 $\mathrm{mm}$, internal diameter $500 \mu \mathrm{m}$, and system volume $392.5 \mu \mathrm{l}$. The solutions were then delivered into the microchannel at the required flow rates, in a continuous segmented flow manner using syringe pumps (KD Scientific). The reactions were carried out while heating to $40{ }^{\circ} \mathrm{C}$ using an oil bath or CEM microwave $(150 \mathrm{~W})$ for the appropriate residence time of 10 minutes. After collecting the output from the reaction, the cooled crude mixture was separated from the perfluorocarbon then evaporated under reduced pressure. The products were isolated by column chromatography on silica gel using an appropriate solvent system.

\section{General procedure for multi-step synthesis via ring closing metathesis (RCM) followed by Heck coupling under segmented flow conditions in microflow}

Stock solutions were prepared in individual Schlenk tubes under inert atmosphere: (A) A solution of diallylamino derivative $(0.1 \mathrm{M}$, either 1: $199.6 \mathrm{mg}, 0.4 \mathrm{mmol}$; or 5: $69.2 \mathrm{mg}, 0.4 \mathrm{mmol})$ in toluene $(4.0 \mathrm{ml})$; (B) A $0.1 \mathrm{M}$ solution of Grubbs catalyst II $(16.9 \mathrm{mg}, 0.02 \mathrm{mmol})$ in toluene $(0.2$ $\mathrm{ml})$; (C) either pure perfluorononane or perfluorodecalin solvent used as the segmenting phase; (D) A $0.004 \mathrm{M}$ solution of $\mathrm{Pd}\left(\mathrm{PPh}_{3}\right)_{4}(46.2 \mathrm{mg}, 0.04 \mathrm{mmol})$ in toluene $(10.0 \mathrm{ml})$; (E) $0.4 \mathrm{M}$ alkene or aryl halide $(0.4 \mathrm{mmol})$, and triethylamine $(40.4 \mathrm{mg}, 0.4 \mathrm{mmol})$ in toluene $(1.0 \mathrm{ml})$. Each solution was loaded individually into gas-tight glass syringes which were then connected onto the multistep microflow system (PTFE) through a designated inlet using a number of T-connectors. Length of tubing: $43700 \mathrm{~mm}$ (section $12000 \mathrm{~mm}$, residence time of 10.0 minutes) + (section $2-41700 \mathrm{~mm}$, residence time of 60 minutes), internal diameter $500 \mu \mathrm{m}$, and system volume $8576 \mu 1$. The solutions were then delivered into the microchannel at the required flow rates, in a continuous segmented flow manner using syringe pumps (KD Scientific). Firstly the RCM of diallylamino moiety was carried out at $40^{\circ} \mathrm{C}$ by pumping solutions (A) along with solution (B) and (C) into the section 1 placed in the CEM microwave cavity. While in section 2 solutions (D) and (E) were introduced then mixed with the cyclised substrate flow from section 1 at $80{ }^{\circ} \mathrm{C}$ using an oil bath to carry out the Heck coupling. The reactions were carried out for the appropriate total residence time of approximately 70 minutes. After collecting the output from the reaction, the cooled crude toluene mixture was separated from the segmenter phase and evaporated under reduced pressure. The purification was carried out using column chromatography using an appropriate solvent system.

$N, N$-Diallyl-2-iodoaniline (1). ${ }^{14}$ Synthesis carried out according to the General Procedure, compound 1 was obtained in $77 \%$ yield as a colourless oil after purification by column chromatography on silica gel using $10 \%$ diethyl ether in hexane. ${ }^{1} \mathrm{H}$ NMR $\left(500 \mathrm{MHz}, \mathrm{CDCl}_{3}\right): \delta=$ $7.90\left(1 \mathrm{H}, \mathrm{dd}, J=2.0,8.0 \mathrm{~Hz}, H_{\mathrm{Ar}}\right), 7.30\left(1 \mathrm{H}, \mathrm{td}, J=2.0,8.0 \mathrm{~Hz}, H_{\mathrm{Ar}}\right), 7.05(1 \mathrm{H}, \mathrm{dd}, J=2.0,8.0$ $\left.\mathrm{Hz}, H_{\mathrm{Ar}}\right), 6.82\left(1 \mathrm{H}, \mathrm{td}, J=2.0,8.0 \mathrm{~Hz}, H_{\mathrm{Ar}}\right), 5.90-5.80\left(2 \mathrm{H}, \mathrm{m}, \mathrm{NCH}_{2} \mathrm{CHCH}_{2}\right), 5.20\left(2 \mathrm{H}, \mathrm{d}, J_{\text {trans }}=\right.$ $\left.17.2 \mathrm{~Hz}, \mathrm{NCH}_{2} \mathrm{CHCHH}_{\text {trans }}\right), 5.13\left(2 \mathrm{H}, \mathrm{d}, J_{\text {cis }}=10.0 \mathrm{~Hz}, \mathrm{NCH}_{2} \mathrm{CHCHH}_{\text {cis }}\right), 3.65(4 \mathrm{H}, \mathrm{d}, J=6.0$ 
$\left.\mathrm{Hz}, \mathrm{NCH}_{2} \mathrm{CHCH}_{2}\right)$ ppm; ${ }^{13} \mathrm{C}$ NMR $\left(100 \mathrm{MHz}, \mathrm{CDCl}_{3}\right): \delta=151.8\left(C_{\mathrm{Ar}} \mathrm{N}\right), 139.9\left(\mathrm{CH}_{\mathrm{Ar}}\right), 134.8$ $\left(\mathrm{NCH}_{2} \mathrm{CHCH}_{2}\right), 128.4\left(\mathrm{CH}_{\mathrm{Ar}}\right), 125.6\left(\mathrm{CH}_{\mathrm{Ar}}\right), 124.2\left(\mathrm{CH}_{\mathrm{Ar}}\right), 117.7\left(\mathrm{NCH}_{2} \mathrm{CHCH}_{2}\right), 100.4\left(C_{\mathrm{Ar}} \mathrm{I}\right)$, $56.1\left(\mathrm{NCH}_{2} \mathrm{CHCH}_{2}\right)$ ppm.

1-(2-Iodophenyl)-2,5-dihydro-1H-pyrrole (2). ${ }^{15}$ Synthesis carried out according to the General Procedure, compound 2 was obtained in $98 \%$ yield as a colourless oil after purification with column chromatography on silica gel using $5 \%$ ethyl acetate in hexane. ${ }^{1} \mathrm{H}$ NMR $(500 \mathrm{MHz}$, $\left.\mathrm{CDCl}_{3}\right): \delta=7.79\left(1 \mathrm{H}, \mathrm{dd}, J=2.0,8.0 \mathrm{~Hz}, H_{\mathrm{Ar}}\right), 7.18\left(1 \mathrm{H}, \mathrm{td}, J=1.2,8.2 \mathrm{~Hz}, H_{\mathrm{Ar}}\right), 6.90(1 \mathrm{H}, \mathrm{dd}, J$ $\left.=1.5,8.2 \mathrm{~Hz}, H_{\mathrm{Ar}}\right), 6.59\left(1 \mathrm{H}, \mathrm{td}, J=1.1,8.0 \mathrm{~Hz}, \mathrm{H}_{\mathrm{Ar}}\right), 5.85\left(2 \mathrm{H}, \mathrm{s}, \mathrm{NCH}_{2} \mathrm{CH}\right), 4.20(4 \mathrm{H}, \mathrm{s}$, $\left.\mathrm{NCH}_{2} \mathrm{CH}\right)$ ppm; ${ }^{13} \mathrm{C} \mathrm{NMR}\left(100 \mathrm{MHz}, \mathrm{CDCl}_{3}\right): \delta=152.0\left(\mathrm{C}_{\mathrm{Ar}} \mathrm{N}\right), 141.3\left(\mathrm{CH}_{\mathrm{Ar}}\right), 128.9\left(\mathrm{CH}_{\mathrm{Ar}}\right)$, 126.6 $\left(\mathrm{NCH}_{2} \mathrm{CH}\right), 123.2\left(\mathrm{CH}_{\mathrm{Ar}}\right), 120.0\left(\mathrm{CH}_{\mathrm{Ar}}\right), 90.8\left(\mathrm{C}_{\mathrm{Ar}} \mathrm{I}\right), 58.36\left(\mathrm{NCH}_{2} \mathrm{CH}\right) \mathrm{ppm}$.

1-(2-Iodophenyl)-1H-pyrrole (3). ${ }^{16}$ Synthesis carried out according to the General Procedure, compound 3 was obtained in traces as a colourless oil after purification by column chromatography on silica gel using 5\% ethyl acetate in hexane. ${ }^{1} \mathrm{H}$ NMR $\left(500 \mathrm{MHz}, \mathrm{CDCl}_{3}\right): \delta=$ $7.96\left(1 \mathrm{H}, \mathrm{dd}, J=1.2,7.6 \mathrm{~Hz}, H_{\mathrm{Ar}}\right), 7.38\left(1 \mathrm{H}, \mathrm{td}, J=1.2,7.6 \mathrm{~Hz}, H_{\mathrm{Ar}}\right), 7.28(1 \mathrm{H}, \mathrm{dd}, J=1.6,7.6$ $\left.\mathrm{Hz}, H_{\mathrm{Ar}}\right), 7.11\left(1 \mathrm{H}, \mathrm{td}, J=1.6,7.6 \mathrm{~Hz}, H_{\mathrm{Ar}}\right), 6.77(2 \mathrm{H}, \mathrm{t}, J=2.0 \mathrm{~Hz}, \mathrm{NCHCH}), 6.30(2 \mathrm{H}, \mathrm{t}, J=2.0$ $\mathrm{Hz} \mathrm{NCHCH})$ ppm; ${ }^{13} \mathrm{C}$ NMR $\left(100 \mathrm{MHz}, \mathrm{CDCl}_{3}\right): \delta=146.0\left(C_{\mathrm{Ar}} \mathrm{N}\right), 139.0\left(\mathrm{CH}_{\mathrm{Ar}}\right), 131.0\left(\mathrm{CH}_{\mathrm{Ar}}\right)$, $129.5\left(\mathrm{CH}_{\mathrm{Ar}}\right), 128.5\left(\mathrm{CH}_{\mathrm{Ar}}\right), 120.8(\mathrm{NCHCH}), 108.0(\mathrm{NCHCH}), 95.0\left(\mathrm{C}_{\mathrm{Ar}} \mathrm{I}\right) \mathrm{ppm}$.

(E)-Methyl 3-(2-(2H-pyrrol-1(5H)-yl)phenyl)acrylate (4). Synthesis carried out according to the General Procedure, compound 4 was obtained in $64 \%$ yield as a colourless oil after purification by column chromatography on silica gel using $15 \%$ diethyl ether in petroleum ether. ${ }^{1} \mathrm{H}$ NMR (500 $\left.\mathrm{MHz}, \mathrm{CDCl}_{3}\right): \delta=8.10(1 \mathrm{H}, \mathrm{d}, J=15.7 \mathrm{~Hz}, \mathrm{ArCH}), 7.29\left(1 \mathrm{H}, \mathrm{dd}, J=1.2,7.6 \mathrm{~Hz}, H_{\mathrm{Ar}}\right), 7.19(1 \mathrm{H}$, td, $\left.J=2.1,7.9 \mathrm{~Hz}, H_{\mathrm{Ar}}\right), 6.85-6.72\left(2 \mathrm{H}, \mathrm{m}, H_{\mathrm{Ar}}\right), 6.15\left(1 \mathrm{H}, \mathrm{d}, J=16.1 \mathrm{~Hz}, \mathrm{CHCO}_{2} \mathrm{Me}\right), 5.80(2 \mathrm{H}$, s, $\left.\mathrm{NCH}_{2} \mathrm{CH}\right), 4.15\left(4 \mathrm{H}, \mathrm{s}, \mathrm{NCH}_{2} \mathrm{CH}\right), 3.69\left(3 \mathrm{H}, \mathrm{s}, \mathrm{CO}_{2} \mathrm{Me}\right) \mathrm{ppm} ;{ }^{13} \mathrm{C} \mathrm{NMR}\left(100 \mathrm{MHz}, \mathrm{CDCl}_{3}\right): \delta=$ $167.0\left(\mathrm{CO}_{2} \mathrm{Me}\right), 161.2\left(\mathrm{C}_{\mathrm{Ar}} \mathrm{N}\right), 140.5(\mathrm{ArCH}), 130.7\left(\mathrm{CH}_{\mathrm{Ar}}\right), 129.6\left(\mathrm{CH}_{\mathrm{Ar}}\right), 127.7\left(\mathrm{CH}_{\mathrm{Ar}}\right), 126.2$ $\left(\mathrm{NCH}_{2} \mathrm{CH}\right), 122.8\left(\mathrm{CH}_{\mathrm{Ar}}\right), 117.0\left(\mathrm{CHCO}_{2} \mathrm{Me}\right), 109.9\left(\mathrm{CH}_{\mathrm{Ar}}\right), 58.8\left(\mathrm{NCH}_{2} \mathrm{CH}\right), 51.6\left(\mathrm{CO}_{2} \mathrm{Me}\right) \mathrm{ppm}$; MS (ES) m/z (\%): $230(100)\left[\mathrm{M}+\mathrm{H}^{+}\right], 231$ (15); HRMS for $\left[\mathrm{M}+\mathrm{H}^{+}\right]$for $\left[\mathrm{C}_{14} \mathrm{H}_{15} \mathrm{NO}_{2}+\mathrm{H}\right]^{+}$: calc. 230.1181, found 230.1185; IR: $v$ 2978, 1963, 1727 (CH=CH-CO), 1444, 1383, 1351, 1297, 1130, 1076, 1043, 934 (trans- $\mathrm{CH}=\mathrm{CH}), 846,794$ (cis- $\mathrm{CH}=\mathrm{CH}) \mathrm{cm}^{-1}$.

$\mathbf{N}, \mathbf{N}$-Diallylaniline (5). ${ }^{17}$ Synthesis carried out according to the General Procedure, compound 5 was obtained in $89 \%$ yield as a colourless oil after purification by column chromatography on silica gel using $10 \%$ diethyl ether in petroleum ether. ${ }^{1} \mathrm{H}$ NMR $\left(500 \mathrm{MHz}, \mathrm{CDCl}_{3}\right): \delta=7.30-7.20$ $\left(2 \mathrm{H}, \mathrm{m}, \mathrm{H}_{\mathrm{Ar}}\right), 6.78-6.69\left(3 \mathrm{H}, \mathrm{m}, \mathrm{H}_{\mathrm{Ar}}\right), 5.89\left(2 \mathrm{H}, \mathrm{m}, \mathrm{NCH}_{2} \mathrm{CHCH}_{2}\right), 5.22\left(2 \mathrm{H}, \mathrm{d}, J_{\text {trans }}=16.3 \mathrm{~Hz}\right.$, $\left.\mathrm{NCH}_{2} \mathrm{CHCHH}_{\text {trans }}\right), 5.14\left(2 \mathrm{H}, \mathrm{d}, J_{\text {cis }}=10.0 \mathrm{~Hz}, \mathrm{CH}_{2} \mathrm{CHCHH}_{\text {cis }}\right), 3.98(4 \mathrm{H}, \mathrm{d}, J=4.9 \mathrm{~Hz}$, $\left.\mathrm{NCH}_{2} \mathrm{CHCH}_{2}\right)$ ppm; ${ }^{13} \mathrm{C} \mathrm{NMR}\left(100 \mathrm{MHz}, \mathrm{CDCl}_{3}\right): \delta=148.7\left(C_{\mathrm{Ar}} \mathrm{N}\right), 134.1\left(\mathrm{NCH}_{2} \mathrm{CHCH}_{2}\right), 129.1$ $\left(\mathrm{CH}_{\mathrm{Ar}}\right), 116.3\left(\mathrm{NCH}_{2} \mathrm{CHCH}_{2}\right), 116.0\left(\mathrm{CH}_{\mathrm{Ar}}\right), 112.4\left(\mathrm{CH}_{\mathrm{Ar}}\right), 52.5\left(\mathrm{NCH}_{2} \mathrm{CHCH}_{2}\right) \mathrm{ppm}$.

1-Phenyl-2,5-dihydro-1H-pyrrole (6). ${ }^{18}$ Synthesis carried out according to the General Procedure, compound 6 was obtained in 96\% yield as a colourless oil after purification by column chromatography on silica gel using $10 \%$ diethyl ether in petroleum ether. ${ }^{1} \mathrm{H}$ NMR $(500 \mathrm{MHz}$, $\left.\mathrm{CDCl}_{3}\right): \delta=7.20\left(2 \mathrm{H}, \mathrm{t}, J=8.0 \mathrm{~Hz}, H_{\mathrm{Ar}}\right), 6.6\left(1 \mathrm{H}, \mathrm{t}, J=7.8 \mathrm{~Hz}, H_{\mathrm{Ar}}\right), 6.45(2 \mathrm{H}, \mathrm{d}, J=7.3 \mathrm{~Hz}$, 
$\left.H_{\mathrm{Ar}}\right), 5.86\left(2 \mathrm{H}, \mathrm{s}, \mathrm{NCH}_{2} \mathrm{CH}\right), 4.00\left(4 \mathrm{H}, \mathrm{s}, \mathrm{NCH}_{2} \mathrm{CH}\right) \mathrm{ppm} ;{ }^{13} \mathrm{C} \mathrm{NMR}\left(100 \mathrm{MHz}, \mathrm{CDCl}_{3}\right): \delta=147.1$ $\left(C_{\mathrm{Ar}} \mathrm{N}\right), 129.4\left(\mathrm{CH}_{\mathrm{Ar}}\right), 126.4\left(\mathrm{NCH}_{2} \mathrm{CH}\right), 115.6\left(\mathrm{CH}_{\mathrm{Ar}}\right), 111.2\left(\mathrm{CH}_{\mathrm{Ar}}\right), 54.5\left(\mathrm{NCH}_{2} \mathrm{CH}\right) \mathrm{ppm}$.

1-Phenyl-1H-pyrrole (7). ${ }^{19}$ Synthesis carried out according to the General Procedure, compound 7 was obtained in traces as a solid after purification by column chromatography on silica gel using $10 \%$ diethyl ether in petroleum ether. ${ }^{1} \mathrm{H} \mathrm{NMR}\left(500 \mathrm{MHz}, \mathrm{CDCl}_{3}\right): \delta=7.48-7.56\left(4 \mathrm{H}, \mathrm{m}, H_{\mathrm{Ar}}\right)$, 7.31-7.35 (1H, m, $\left.H_{\mathrm{Ar}}\right), 7.21(2 \mathrm{H}, \mathrm{t}, J=2.0 \mathrm{~Hz}, \mathrm{NCHCH}), 6.50(2 \mathrm{H}, \mathrm{t}, J=2.0 \mathrm{~Hz}, \mathrm{NCHCH}) \mathrm{ppm}$; ${ }^{13} \mathrm{C}$ NMR $\left(100 \mathrm{MHz}, \mathrm{CDCl}_{3}\right): \delta=139.7\left(C_{\mathrm{Ar}} \mathrm{N}\right), 129.5\left(\mathrm{CH}_{\mathrm{Ar}}\right), 126.0(\mathrm{NCHCH}), 121.3\left(\mathrm{CH}_{\mathrm{Ar}}\right)$, $118.1\left(\mathrm{CH}_{\mathrm{Ar}}\right), 111.6(\mathrm{NCHCH}) \mathrm{ppm}$.

1,3-Diphenyl-1H-pyrrole (8). ${ }^{20}$ Synthesis carried out according to the General Procedure, compound $\mathbf{8}$ was obtained in $60 \%$ yield as a colourless solid after purification by column chromatography on silica gel using $10 \%$ diethyl ether in petroleum ether. mp $119-121{ }^{\circ} \mathrm{C}$ (lit. mp $\left.118-119{ }^{\circ} \mathrm{C}\right) ;{ }^{1} \mathrm{H}$ NMR $\left(500 \mathrm{MHz}, \mathrm{CDCl}_{3}\right): \delta=7.61\left(2 \mathrm{H}, \mathrm{d}, J=8.1 \mathrm{~Hz}, H_{\mathrm{Ar}}\right), 7.49-7.19(5 \mathrm{H}, \mathrm{m}$, $\left.H_{\mathrm{Ar}}\right), 7.34\left(2 \mathrm{H}, \mathrm{t}, J=7.6 \mathrm{~Hz}, H_{\mathrm{Ar}}\right), 7.25\left(1 \mathrm{H}, \mathrm{t}, J=7.9 H_{\mathrm{Ar}}\right), 7.15(1 \mathrm{H}, \mathrm{dd}, J=1.1,2.8 \mathrm{~Hz}$, $\mathrm{NCHCPh}), 6.85(1 \mathrm{H}, \mathrm{dd}, J=1.6,4.1 \mathrm{~Hz}, \mathrm{NCHCH}), 6.39(1 \mathrm{H}, \mathrm{dd}, J=2.8,4.1 \mathrm{~Hz}, \mathrm{NCHCH}) \mathrm{ppm}$; ${ }^{13} \mathrm{C}$ NMR $\left(100 \mathrm{MHz}, \mathrm{CDCl}_{3}\right): \delta=146.5\left(C_{\mathrm{Ar}} \mathrm{N}\right), 138.5\left(C_{\mathrm{Ar}} \mathrm{CCH}\right), 138.0\left(\mathrm{CH}_{\mathrm{Ar}}\right), 129.6\left(\mathrm{C}_{\mathrm{Ar}} \mathrm{CCH}\right)$, $128.9\left(\mathrm{CH}_{\mathrm{Ar}}\right), 128.8\left(\mathrm{CH}_{\mathrm{Ar}}\right), 127.2\left(\mathrm{CH}_{\mathrm{Ar}}\right), 126.6\left(\mathrm{CH}_{\mathrm{Ar}}\right), 126.2\left(\mathrm{CH}_{\mathrm{Ar}}\right), 122.3(\mathrm{NCHCH}), 111.2$ $(\mathrm{NCHCPh}), 100.0(\mathrm{NCHCH}) \mathrm{ppm}$.

$N, N$-Diallyl-1,2-phenylenediamine. ${ }^{21}$ Synthesis carried out according to the General Procedure, $\mathrm{N}, \mathrm{N}$-diallyl-1,2-phenylenediamine was obtained in $40 \%$ yield as a colourless oil after purification by medium pressure liquid chromatography on silica gel using $2 \%$ diethyl ether in hexane. ${ }^{1} \mathrm{H}$ NMR $\left(500 \mathrm{MHz}, \mathrm{CDCl}_{3}\right): \delta=7.02\left(1 \mathrm{H}, \mathrm{dd}, J=1.4,8.0 \mathrm{~Hz}, H_{\mathrm{Ar}}\right), 6.94(1 \mathrm{H}, \mathrm{td}, J=1.5,8.0 \mathrm{~Hz}$, $\left.H_{\mathrm{Ar}}\right), 6.75\left(2 \mathrm{H}, \mathrm{m}, \mathrm{H}_{\mathrm{Ar}}\right), 5.90-5.75\left(2 \mathrm{H}, \mathrm{m}, \mathrm{NCH}_{2} \mathrm{CHCH}_{2}\right), 5.20\left(2 \mathrm{H}, \mathrm{d}, J_{\text {trans }}=17.0, \mathrm{~Hz}\right.$, $\left.\mathrm{NCH}_{2} \mathrm{CHCHH}_{\text {trans }}\right), 5.17\left(2 \mathrm{H}, \mathrm{d}, J_{\text {cis }}=12.0 \mathrm{~Hz}, \mathrm{NCH}_{2} \mathrm{CHCHH}_{\text {cis }}\right), 4.08\left(2 \mathrm{H}, \mathrm{br}, \mathrm{NH}_{2}\right), 3.58(4 \mathrm{H}, \mathrm{d}$, $\left.J=6.0 \mathrm{~Hz}, \mathrm{NCH}_{2} \mathrm{CHCH}_{2}\right) \mathrm{ppm} ;{ }^{13} \mathrm{C} \mathrm{NMR}\left(100 \mathrm{MHz}, \mathrm{CDCl}_{3}\right): \delta=142.6\left(C_{\mathrm{Ar}_{\mathrm{NAllyl}}}\right), 137.2$ $\left(C_{\mathrm{Ar} H}\right), \quad 135.2 \quad\left(\mathrm{NCH}_{2} \mathrm{CHCH}_{2}\right), \quad 124.7 \quad\left(\mathrm{CH}_{\mathrm{Ar}}\right), \quad 122.7 \quad\left(\mathrm{CH}_{\mathrm{Ar}}\right), \quad 117.9 \quad\left(\mathrm{CH}_{\mathrm{Ar}}\right), \quad 117.2$ $\left(\mathrm{NCH}_{2} \mathrm{CHCH}_{2}\right), 115.2\left(\mathrm{CH}_{\mathrm{Ar}}\right), 55.3\left(\mathrm{NCH}_{2} \mathrm{CHCH}_{2}\right)$ ppm.

tert-Butyl-2-(diallylamino)-phenylcarbamate (9). $N, N$-Diallyl-1,2-phenylenediamine (1.0 g, 5.32 $\mathrm{mmol})$ and di-t-butyldicarbonate $(1.39 \mathrm{~g}, 6.4 \mathrm{mmol})$ were dissolved in dichloromethane $(25 \mathrm{ml})$ with triethylamine $(0.80 \mathrm{~g}, 7.9 \mathrm{mmol})$. The mixture was stirred for $12 \mathrm{~h}$ at room temperature. The mixture was washed with brine $(25 \mathrm{ml})$ followed by water $(25 \mathrm{ml})$, and the organic layer was dried with magnesium sulfate and then concentrated under reduced pressure. The resulting crude reaction mixture was purified using silica gel column chromatography (5\% diethyl ether in hexane) to obtain 9 (1.32 g, $4.57 \mathrm{mmol}, 86 \%)$ as a colourless oil. ${ }^{1} \mathrm{H}$ NMR $\left(500 \mathrm{MHz}, \mathrm{CDCl}_{3}\right): \delta=7.71$ $\left(1 \mathrm{H}, \mathrm{br}, H_{\mathrm{Ar}}\right), 7.13-7.08\left(2 \mathrm{H}, \mathrm{m}, \mathrm{H}_{\mathrm{Ar}}\right), 6.95\left(1 \mathrm{H}, \mathrm{m}, \mathrm{H}_{\mathrm{Ar}}\right), 5.85-5.72\left(2 \mathrm{H}, \mathrm{m}, \mathrm{NCH}_{2} \mathrm{CHCH}_{2}\right), 5.10$ $\left(2 \mathrm{H}, \mathrm{d}, J_{\text {trans }}=17.2 \mathrm{~Hz}, \mathrm{NCH}_{2} \mathrm{CHCHH}_{\text {trans }}\right), 5.05\left(2 \mathrm{H}, \mathrm{d}, J_{\text {cis }}=10.0 \mathrm{~Hz}, \mathrm{NCH}_{2} \mathrm{CHCHH}_{\text {cis }}\right), 3.5(4 \mathrm{H}$, $\left.\mathrm{d}, J=6.0 \mathrm{~Hz}, \mathrm{NCH}_{2} \mathrm{CH}\right), 1.54\left(9 \mathrm{H}, \mathrm{s}, \mathrm{OCMe}_{3}\right) \mathrm{ppm} ;{ }^{13} \mathrm{C} \mathrm{NMR}\left(100 \mathrm{MHz}, \mathrm{CDCl}_{3}\right): \delta=153.0$ $\left(\mathrm{NCO}_{2} \mathrm{CMe}_{3}\right), 147.0\left(C_{\mathrm{Ar} \mathrm{NAllyl}}\right), 135.2\left(C_{\mathrm{Ar}} \mathrm{NHCO}_{2} \mathrm{CMe}_{3}\right), 134.4\left(\mathrm{NCH}_{2} \mathrm{CHCH}_{2}\right), 125.4\left(\mathrm{CH}_{\mathrm{Ar}}\right)$, $122.9\left(\mathrm{CH}_{\mathrm{Ar}}\right), \quad 121.8\left(\mathrm{CH}_{\mathrm{Ar}}\right), \quad 118.0\left(\mathrm{CH}_{\mathrm{Ar}}\right), \quad 117.8\left(\mathrm{NCH}_{2} \mathrm{CHCH}_{2}\right), \quad 80.1 \quad\left(\mathrm{OCMe}_{3}\right), \quad 56.7$

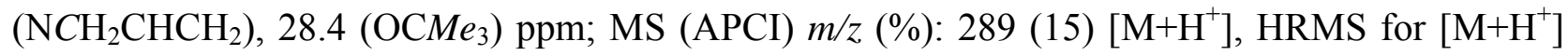


for $\left[\mathrm{C}_{17} \mathrm{H}_{24} \mathrm{~N}_{2} \mathrm{O}_{2}+\mathrm{H}\right]^{+}$: calc. 289.1916, found 289.1922; IR: v 3367, $3078\left(\mathrm{CH}=\mathrm{CH}_{2}\right), 2979\left(\mathrm{CH}_{2}\right)$, 1810, $1727.9\left(\mathrm{NCO}_{2} \mathrm{tBu}\right), 1643\left(\mathrm{CH}=\mathrm{CH}_{2}\right), 1591,1516,1448,1219,1157,1070,923 \mathrm{~cm}^{-1}$.

tert-Butyl 2-(2H-pyrrol-1(5H)-yl)-phenylcarbamate (10). Synthesis carried out according to the General Procedure, compound $\mathbf{1 0}$ was obtained in 91\% yield as a colourless oil after purification by column chromatography on silica gel using $20 \%$ diethyl ether in petroleum ether. ${ }^{1} \mathrm{H}$ NMR (500 $\left.\mathrm{MHz}, \mathrm{CDCl}_{3}\right): \delta=7.41\left(1 \mathrm{H}, \mathrm{br}, H_{\mathrm{Ar}}\right), 7.09\left(1 \mathrm{H}, \mathrm{dd}, J=1.2,7.6 \mathrm{~Hz}, H_{\mathrm{Ar}}\right), 6.97(1 \mathrm{H}, \mathrm{td}, J=2.1,7.9$ $\left.\mathrm{Hz}, H_{\mathrm{Ar}}\right), 6.90\left(1 \mathrm{H}, \mathrm{td}, J=2.4,8.3 \mathrm{~Hz}, \mathrm{H}_{\mathrm{Ar}}\right), 5.82\left(2 \mathrm{H}, \mathrm{s}, \mathrm{NCH}_{2} \mathrm{CH}\right), 3.92\left(4 \mathrm{H}, \mathrm{s}, \mathrm{NCH}_{2} \mathrm{CH}\right), 1.43$ $\left(9 \mathrm{H}, \mathrm{s}, \mathrm{OCMe}_{3}\right) \mathrm{ppm} ;{ }^{13} \mathrm{C} \mathrm{NMR}\left(100 \mathrm{MHz}, \mathrm{CDCl}_{3}\right): \delta=153.5\left(\mathrm{NCO}_{2} \mathrm{CMe}_{3}\right), 140.9\left(C_{\mathrm{Ar}} \mathrm{NC}_{4} \mathrm{H}_{6}\right)$, $134.0\left(C_{\mathrm{Ar}} \mathrm{NCO}_{2} \mathrm{CMe}_{3}\right), 126.9\left(\mathrm{NCH}_{2} \mathrm{CH}\right), 124.1\left(\mathrm{CH}_{\mathrm{Ar}}\right), 123.4\left(\mathrm{CH}_{\mathrm{Ar}}\right), 121.3\left(\mathrm{CH}_{\mathrm{Ar}}\right), 119.9$

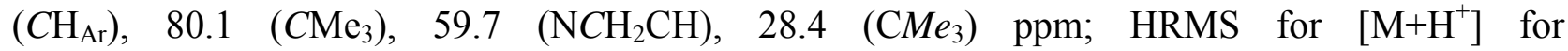
$\left[\mathrm{C}_{15} \mathrm{H}_{20} \mathrm{~N}_{2} \mathrm{O}_{2}+\mathrm{H}\right]^{+}$: calc. 261.1603, found 261.1594; IR: $v$ 3362, 2977, $2867\left(\mathrm{NCH}_{2}\right), 1729$ $\left(\mathrm{NCO}_{2} \mathrm{Bu}\right), 1594,1517,1158,832,747$ (cis- $\left.\mathrm{CH}=\mathrm{CH}\right) \mathrm{cm}^{-1}$.

tert-Butyl-2-(1H-pyrrol-1-yl)-phenylcarbamate (11). Synthesis carried out according to the General Procedure, compound 11 was obtained in 32\% yield as a colourless oil after purification by column chromatography on silica gel using $20 \%$ diethyl ether in petroleum ether. ${ }^{1} \mathrm{H}$ NMR $(500$ $\left.\mathrm{MHz}, \mathrm{CDCl}_{3}\right): \delta=8.12\left(1 \mathrm{H}, \mathrm{dd}, J=1.2,7.6 \mathrm{~Hz}, H_{\mathrm{Ar}}\right), 7.28\left(1 \mathrm{H}, \mathrm{td}, J=1.2,7.6 \mathrm{~Hz}, H_{\mathrm{Ar}}\right), 7.12(1 \mathrm{H}$, $\left.\mathrm{dd}, J=1.6,7.6 \mathrm{~Hz}, H_{\mathrm{Ar}}\right), 6.90\left(1 \mathrm{H}, \mathrm{td}, J=1.6,7.6 \mathrm{~Hz}, H_{\mathrm{Ar}}\right), 6.71(2 \mathrm{H}, \mathrm{t}, J=2.0 \mathrm{~Hz}, \mathrm{NCHCH})$, $6.32(2 \mathrm{H}, \mathrm{t}, J=2.0 \mathrm{~Hz}, \mathrm{NCHCH}), 1.41\left(9 \mathrm{H}, \mathrm{s}, \mathrm{OCMe}_{3}\right) \mathrm{ppm} ;{ }^{13} \mathrm{C} \mathrm{NMR}\left(100 \mathrm{MHz}, \mathrm{CDCl}_{3}\right): \delta=$ $152.6\left(\mathrm{NCO}_{2} \mathrm{CMe}_{3}\right), 134.1\left(C_{\mathrm{Ar}} \mathrm{NC}_{4} \mathrm{H}_{4}\right), 129.9\left(C_{\mathrm{Ar}} \mathrm{NCO}_{2} \mathrm{CMe}_{3}\right), 128.6\left(\mathrm{CH}_{\mathrm{Ar}}\right), 126.9\left(\mathrm{CH}_{\mathrm{Ar}}\right)$, $122.8\left(\mathrm{CH}_{\mathrm{Ar}}\right), 122.0(\mathrm{NCHCH}), 119.8\left(\mathrm{CH}_{\mathrm{Ar}}\right), 110.2(\mathrm{NCHCH}), 80.9\left(\mathrm{CMe}_{3}\right), 28.3\left(\mathrm{CMe}_{3}\right) \mathrm{ppm}$; HRMS for $\left[\mathrm{M}^{+}\right]$for $\left[\mathrm{C}_{15} \mathrm{H}_{18} \mathrm{~N}_{2} \mathrm{O}_{2}\right]^{+}$: calc. 258.1368, found 258.1374; IR: $v$ 3413, 3054, 2985, 2305 , $1727\left(\mathrm{NCO}_{2} \mathrm{Bu}\right), 1596,1523,1483,1450,1265(\mathrm{NCH}=\mathrm{CH}), 1158,896,739(\mathrm{NCH}=\mathrm{CH}) \mathrm{cm}^{-1}$.

\section{Acknowledgements}

We thank EPSRC for support of this work and the EPSRC National Mass Spectrometry Service Centre, Swansea, for mass spectrometric data.

\section{References}

1. (a) Microreactors in Organic Synthesis and Catalysis, Wirth, T., Ed. Wiley-VCH: Weinheim, 2008. (b) Yoshida, J.; Nagaki, A.; Yamada, T. Chem. Eur. J. 2008, 14, 7450; (c) Watts, P.; Wiles, C. Org. Biomol. Chem. 2007, 5, 727. (d) Ahmed-Omer, B.; Brandt, J. C.; Wirth, T. Org. Biomol. Chem. 2007, 5, 733. (e) Mason, B. P.; Price, K. E.; Steinbacher, J. L.; Bogdan, A. R.; McQuade D. T. Chem. Rev. 2007, 107, 2300.

2. Ahmed-Omer, B.; Barrow, D. A.; Wirth, T. Tetrahedron Lett. 2009, 50, 3352.

3. Solodenko, W.; Wen, H.; Leue, S.; Stuhlmann, F.; Sourkouni-Argirusi, G.; Jas, G.; Schönfeld, H.; Kunz, U.; Kirschning, A. Eur. J. Org. Chem. 2004, 3601. 
4. Karbass, N.; Sans, V.; Garcia-Verdugo, E.; Burguete, M. I.; Luis, S. V. Chem. Commun. 2006, 3095.

5. Nikbin, N.; Ladlow, M.; Ley, S. V. Org. Process Res. Dev. 2007, 11, 458.

6. Snyder, D. A.; Noti, C.; Seeberger, P. H.; Schael, F.; Bieber, T.; Rimmel, G.; Ehrfeld, W. Helv. Chim. Acta 2005, 88, 1.

7. Murphy, E. R.; Martinelli, J. R.; Zaborenko, N.; Buchwald, S. L.; Jensen, K. F. Angew. Chem. Int. Ed. 2007, 46, 1734.

8. McMullen, J. P.; Stone, M. T.; Buckwald, S. L.; Jensen, K. F. Angew. Chem. Int. Ed. 2010, 49, 7076.

9. Singh, B. K.; Kaval, N.; Tomar, S.; Van der Eycken, E.; Parmar V. S. Org. Process Res. Dev. 2008, 12, 468-474.

10. Harries, N.; Burns, J. R.; Barrow, D. A.; Ramshaw, C. J. Heat Mass Transf. 2003, 46, 3313.

11. Bhanage, B. M.; Zhao, F.-G.; Shirai, M.; Arai, M. Tetrahedron Lett. 1998, 39, 9509.

12. (a) Liu, S.; Fukuyama, T.; Sato, M.; Ryu, I. Synlett 2004, 1814; (b) Liu, S.; Fukuyama, T.; Ryu, I. Org. Process Res. Dev. 2004, 8, 477.

13. (a) Schwab, P.; Grubbs, R. H.; Ziller, J. W. J. Am. Chem. Soc. 1996, 118, 100; (b) Miller, S. J.; Blackwell, H. E.; Grubbs, R. H. J. Am. Chem. Soc. 1996, 118, 9606; (c) Scholl, M.; Ding, S.; Woo Lee, C.; Grubbs, R. H. Org. Lett. 1999, 1, 953; (d) Saito, N.; Sato, Y.; Mori, M. Org. Lett. 2002, 4, 803.

14. Molander, G. A.; Harring, L. S. J. Org. Chem. 1990, 55, 6171.

15. Evans, P.; Grigg, R.; Monteith, M. Tetrahedron Lett. 1999, 40, 5247.

16. Campo, M. A.; Larock, R. C. J. Org. Chem. 2002, 67, 5616.

17. Pace, V.; Martínez, F.; Fernández, M.; Sinisterra, J. V.; Alcántara, A. R. Org. Lett. 2007, 9, 2661.

18. Bobbitt, J. M.; Amundsen, L. H.; Steiner, R. I. J. Org. Chem. 1960, 25, 2230.

19. Zhu, L.; Cheng, L.; Zhang, Y.; Xie, R.; You, J. J. Org. Chem. 2007, 72, 2737.

20. Kagabu, S.; Tsuji, H.; Kawai, I.; Ozeki, H. Bull. Chem. Soc. Jpn. 1995, 68, 341.

21. Ding, B.; Teng, Z.; Keese, R. J. Org. Chem. 2002, 67, 8906. 